Commun: Communication et organisation

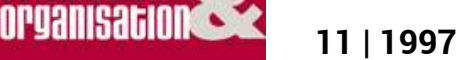

Négociation et médiation dans l'entreprise

\title{
Le médiateur dans le monde : un sujet passionnel
}

\section{Martine Versel}

\section{OpenEdition}

Journals

Édition électronique

URL : http://journals.openedition.org/communicationorganisation/1927

DOI : 10.4000/communicationorganisation. 1927

ISSN : 1775-3546

\section{Éditeur}

Presses universitaires de Bordeaux

\section{Édition imprimée}

Date de publication : 1 mai 1997

ISSN : 1168-5549

\section{Référence électronique}

Martine Versel, «Le médiateur dans le monde : un sujet passionnel », Communication et organisation

[En ligne], 11 | 1997, mis en ligne le 26 mars 2012, consulté le 20 avril 2019. URL : http://

journals.openedition.org/communicationorganisation/1927 ; DOI : 10.4000/

communicationorganisation. 1927

Ce document a été généré automatiquement le 20 avril 2019.

(c) Presses universitaires de Bordeaux 


\title{
Le médiateur dans le monde : un sujet passionnel
}

\author{
Martine Versel
}

1 À partir de deux articles ${ }^{1}$ du journal Le Monde datés du (02/04/1994), nous aborderons ce que la notion de médiateur pose lors de sa mise en discours. Notre méthodologie empruntera des outils appartenant à la sémiotique.

2 Le postulat affiché de la sémiotique n'est pas tant l'étude de la communication que celle de la signification, au plan de l'énoncé (syntaxe et sémantique) et de l'énonciation (ordre de la pragmatique qui met en jeu les conditions de la production, le contexte et les rapports d'interlocution). Notre approche est donc davantage liée à la linguistique qu'à l'anthropologie ou à la sociologie même s'il y eut au cours de l'évolution de la discipline des correspondances entre elles. Nous ne citerons que deux auteurs, l'anthropologue C. Levi Strauss ou P. Bourdieu chez les «nouveaux sociologues ».

3 Par ailleurs, consciente de ses limites, la sémiotique s'intéresse à ce qu'il est convenu d'appeler la signification primaire (le minimum de compréhension effective selon les propres mots du sémioticien J. Courtés), laissant le soin aux nombreuses autres disciplines d'avoir une approche interprétative (la signification secondaire de nature encyclopédique au sens de U. Eco).

4 Nous nous intéresserons dans cette étude à un aspect discursif particulier du médiateur : son agencement thymique ${ }^{2}$. Il s'agit de ce que l'on pourrait finalement appeler le paraître de l'être du médiateur.

5 Si nous voulions, en premier lieu condenser le parcours proposé par ces articles, nous dirions qu'ils définissent le médiateur en définissant tout d'abord la médiation.

6 Il est également aisé de se rendre compte à la lecture de cet énoncé, que le rôle de médiateur journalistique consiste à mettre en place une stratégie cognitive assimilable au rôle thématique de la vigilance: ne pas se laisser prendre aux pièges de l'apparence, de l'ordre du paraître, qui doit aussi être sanctionné au plan de l'être.

7 Il convient d'ajouter que l'énonciation met en jeu un faire persuasif de l'énonciateur, il lui revient de faire croire, appelant ainsi le faire interprétatif d'un énonciataire (le lecteur du 
Monde) qui par définition, et grâce à son statut de lecteur - croit - par la médiation de l'objet même (le journal).

On voit là une juxtaposition discursive : un discours qui transite, pour ainsi dire d'une instance à l'autre selon les modalités épistémiques (croire) et véridictoires (paraître/ être), variables eu égard aux deux instances en jeu dans la définition : la médiation, et le médiateur dans le journal Le Monde.

9 Cependant, dans l'extrait de presse que nous avons choisi nous montrerons la prégnance de la catégorie thymique ${ }^{3}$ dans la constitution de ce sujet particulier du discours.

Tout se passe comme si le médiateur voulait masquer l'instance du faire croire inhérente à toute ligne de rédaction, qu'il soit manipulateur (relation factitive, faire-faire) ou judicateur (être/paraître), dans le développement discursif d'une dimension thymique, pour finalement effacer, en partie du moins, la trame narrative manipulatoire.

11 Nous tenterons donc de saisir ces éléments thymiques, en respectant une donnée épistémologique de la théorie sémiotique posant que tout énoncé de faire est surdéterminé par une ou plusieurs modalités de l'être.

\section{De la médiation au médiateur : une disposition passionnelle naturelle?}

12 Nous proposons tout d'abord de préciser ce que nous entendons par disposition pathémique.

13 Il est question pour nous de la trajectoire existentielle du sujet dans le discours, c'est-àdire la logique interne au sujet. AJ. Greimas cite pour expliquer cette notion, la différence entre l'économe et l'avare. Si la compétence de l'économe est actualisée en situation, celle de l'avare se manifeste même si la situation ne s'y prête plus. Le rôle thémathique est itératif alors que le rôle pathémique est permanent. ${ }^{4}$

«Personne qui s'entremet pour faciliter un accord entre deux ou plusieurs personnes. »

Nous venons de citer ci-dessus la définition du terme médiateur du Petit Robert.

L'analyse révèle alors une relation actantielle entre le médiateur, sujet de faire, et un objet de valeur « conformité de pensée » eu égard à la définition de " accord ».

Il s'agit là d'une conjonction forte, marque de cohésion, au niveau de l'énoncé. Il n'y a aucun effet de sens de dispersion, la trajectoire narrative est unilatérale sans que l'on saisisse une polarité centrée soit sur le sujet désirant (le médiateur), soit sur l'objet de valeur « conformité de pensée », objet désiré. Nous précisons que les "personnes » dans la définition ci-dessus occupent au plan de l'énoncé le statut narratif de sujets d'état dont l'existence sémiotique n'est posée que par la relation orientée, par la jonction avec l'objet de valeur.

Nous nous trouvons dans une dynamique de l'agir, c'est-à-dire dans la transformation d'un état en un autre état, présupposant en terme de contrat de communication une relation fiduciaire sur laquelle repose la tension du discours. Le discours d'usage n'en précise pas les valeurs sémantiques, mais on peut imaginer qu'il s'agirait d'une gradualité dont les polarités extrêmes seraient peut-être la confiance et l'autorité. 
19 En sémiotique de l'énonciation le médiateur appartiendrait à la catégorie des énonciateurs délégués susceptibles de transmettre le discours d'une instance transcendante.

Pour procéder à l'analyse, mais plus encore, pour respecter le contenu, nous le découperons en deux séquences correspondant aux deux articles cités ci-dessus qui suivent un agencement énonciatif spécifique. L'on passe dans le premier article d'un protoénonciateur, dont la figure du discours est essentiellement la médiation à celui du médiateur, dans le deuxième article.

ne parle jamais en son nom propre. Il existe comme représentant, porte-parole de la rédaction du Monde. Nous citons l'article : « Le rôle du médiateur ${ }^{5}$ »

"Nous allons nous y employer. Nous, c'est-à-dire tousceux qui font ce journal et tous ceux qui...»

« Dans la pratique le médiateur sera donc l'interlocuteur privilégié... »

On pourrait alors hâtivement associer les notions de médiation et de médiateur à celle de porte-parole. Celle-ci est liée comme le laisse entendre les citations ci-dessus au concept de délégation proposé par $\mathrm{P}$. Bourdieu, qui relient explicitement le rôle de porte-parole à la notion d'autorité. Reprenons le texte :

26 "et réciproquement, le porte-parole des journalistes pour affirmer leur bon droit, exposer leurs difficultés, les contraintes, et les limites de leur travail, reconnaître leurs erreurs et en débattre franchement. »

Le réseau déictique du contenu, tant par la mise en énoncé d'un protoénonciateur dans la première séquence, que dans l'utilisation des déictiques "nous", «tous", dans la deuxième séquence laisse penser que nous tenons là le stéréotype du porte-parole, c'està-dire non seulement l'autorité que nous avons évoquée mais également la modestie qu'illustre préférentiellement le premier article considéré. Il concourt à l'émaillage au sein du discours d'une force argumentative certaine et participe comme nous l'avons déjà avancé à une trame narrative d'ordre manipulatoire, à la fois nécessaire à l'installation d'un faire croire, et propre à faire valider par le lecteur sa visée narrative : la nécessité « naturelle » de la médiation et du médiateur.

Il y a aussi une tension modale qui s'inscrit sur l'axe du /devoir/ :

Première séquence :

«trouver naturellement sa place dans la page courrier... »

« traiter les questions que se posent les journalistes sur leur travail et aussi celles que leur renvoient les lecteurs.»

Or, une des caractéristiques de la mise en discours du médiateur dans les colonnes du Monde, réside dans une ordonnance pathémique qui tend à effacer les "arêtes " argumentatives inhérentes au statut du médiateur, au profit d'un autre effet de sens, celui de l'ouverture et de l'attente. Notre travail d'analyse suivra dès lors les deux séquences que nous avons précédemment posées et s'emploiera à exposer justement ses effets de sens propres à la mise en discours du médiateur. 


\section{Analyse de la première séquence (Le rendez-vous du Médiateur) :}

Au-delà du contrat de manipulation mise en place grâce à un réseau argumentant, notamment par les positions d'énonciateur délégué et les traces épistémiques qui les relaient au plan narratif; nous avons la mise en discours, dans ce segment de la chaîne discursive, d'un contraste entre un programme et un anti-programme: "qu'elles se recoupent... »

"Qu'elles s'opposent... on ne saurait pas les laisser en l'état »

"Qu'elles se recoupent, ou qu'elles s'opposent, elles révèlent des tensions, des incompréhensions, des malentendus des désaccords qu'on ne saurait laisser en l'état. », nous ne sommes pas très loin du programme narratif proposé par le discours d'usage.

La lecture du premier article met de plus en place un objet modal : «la transparence», inscrit dans un programme narratif d'usage présupposant le /faire être/ de la médiation: «...mieux vaut s'en expliquer franchement, en s'appliquant la transparence que Le Monde exige volontiers des autres. Le médiateur s'y emploiera de son mieux. »

7 Nous remarquons par ailleurs dans le sillage de la "transparence » une succession de sémèmes qui créent au niveau de l'énoncé, dans le contexte de la lexie, une isotopie que nous allons préciser. Citons d'abord les sémèmes :

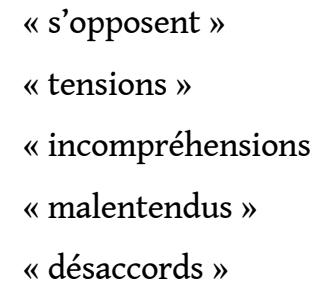

Telle une coupe dans ce «nuage» discursif, il se dessine une isotopie. En effet, chaque occurence citée ci-dessus met en place des unités de sens qui sont actualisées dans le contexte de la lexie.

Il semble qu'il y ait ici un sème qui se propage au sein de ce réseau sémantique, sème isotopant qui serait de l'ordre de l'/entrave/, de l'/obstruction/.

45 De plus, nous avons noté la valeur narrative du sémème «transparence » qui, comme l'indique son sens marque en outre la présence d'un sème qui s'oppose justement à celui de l'/obstruction/. C'est celui de la /circulation/ ou du /passage/. A partir du lexème «netteté » inclu dans cette définition : nous décelons le sème de la /clarté/. peut alors, par propagation rétroactive, considérer l'importance du virtuème /clarté/ qui s'oppose à l'/obscurité/, déterminant alors un deuxième sème isotopant par un processus de contamination.

4 Le parcours sémantique de ce segment textuel s'étend en quelque sorte de l'/obscurité/ à la /clarté/.

48 Dans le deuxième paragraphe de cet article il y a redondance des sèmes de /passage/ et de /lumière/ qui caractérisent au niveau narratif le passage de la médiation au médiateur :

49 «il précise...»

«il conçoit cet échange... » 


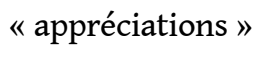

Il est à présent nécessaire pour rendre compte de la cohérence de l'énoncé de procéder à une homologation entre le niveau narratif, (plus précisément avec l'anti programme narratif) que nous rappelons: "que l'on ne saurait laisser en l'état... », et le niveau sémantique : /obscurité/, /lumière/.

Nous nous appuyerons, à ces fins, sur les travaux de C. Zilberberg : Raisons et poétique du sens, Paris, PUF 1988.

Nous citons l'auteur :

- « à l'anti programme narratif, à l'arrêt, nous ferons correspondre un faire émissif »

- «tandis qu'au programme, à l'arrêt de l'arrêt; nous ferons correspondre un faire rémissif que l'on pourrait bien dire aussi continuatif. $»^{6}$

L'auteur précise les valeurs associées à ces deux fonctifs. Le faire émissif (entrainement), et le faire rémissif (inhibition ; stase).

Ce palier missif est un heu médian entre la syntaxe tensive, première indication thymique du sujet percevant et la syntaxe narrative.

On peut, pour revenir à notre énoncé dire alors que la médiation (occupant les deux premiers paragraphes de cette séquence) pose la rupture d'un faire rémissif: «on ne saurait laisser en l'état..." s'appuyant sur les sèmes isotopants /obstruction/, / obscurité/, et qu'elle assume le passage - isotopant /circulation/ -, faire émissif, marqué par la détention, l’ouverture : «...en s'appliquant la transparence... Le médiateur s'y emploiera de son mieux. Il le fera en répondant personnellement à tous ceux... »

2 Nous avons vu la mise en discours d'un sujet percevant. La perception dessine l'esquisse des modalisations qui sont le devoir, le vouloir, le pouvoir et le savoir. Elles rendent signifiantes les tensions perçues.

Nous reprendrons la typologie et la syntaxe modale dérivées des modulations tensives ${ }^{7}$

Modulations ponctuantes Modulations cursives

DEVOIR POUVOIR

\section{SAVOIR VOULOIR}

Modulations clôturantes Modulations ouvrantes

La médiation se situerait au niveau des modulations ponctuantes, préfigurant le devoir et plus essentiellement le /devoir être/ et le médiateur se placerait au niveau des modulations ouvrantes : le vouloir.

Nous allons aborder dans la deuxième séquence le vouloir et plus exactement le /le vouloir être/, afin de déterminer de quelle manière il régit la figure du médiateur, articulant un ensemble pathémique mettant ainsi à distance l'ordre cognitif de la syntaxe narrative.

\section{Analyse de la deuxième séquence (L'avis du médiateur : l'échange)}

Le titre est en soi la condensation du plan narratif de l'article en question. Il pose en effet, un contrat de communication, qui présuppose une modalité précise, celle du / vouloir 
être / comme le confirme en fin d'article l'extrait qui suit: "Avec l'espoir que cet échange, car il s'agit bien de cela, s'approfondira en devenant un rendez-vous naturel. » pathémique régi par une simple volition soutenue par une image but : l'attente, dont le réseau sémantique propre s'apparente au /passage/, voire même à la /lumière/. 
Ce sujet perceptif qu'est le médiateur s'enchâsse, nous l'avons vu, dans une énonciation d'ordre manipulatoire. L'effet de sens consiste donc à mettre à distance de l'énonciataire (le lecteur du « Monde »), cette manipulation.

Il n'est pas de notre ressort d'avancer des interprétations. Cependant, on pourrait dire que, contrairement au porte-parole qui se situe au plan discursif dans l'ordre de l'autorité et de la modestie, le médiateur est une figure en apparence plus simple. Puisqu'il est mu par l'attente et la modulation de l'ouverture confirmée par l'analyse sémantique (/ passage/), il ne subit aucun désordre interne. Or, la spécificité et peut-être même la complexité de sa position discursive résident de façon originale dans la mise à distance des effets de sens manipulatoires du discours par la préhéminence d'une mise en discours du ressenti du médiateur. La présence du sujet percevant chevauche alors, jusqu'à l'évincer, le sujet de faire tel que le pose la définition d'usage généralement associée au médiateur.

\section{Annexe}

\section{Le rendez-vous du médiateur (02/04/1994)}

86 « la première intervention publique du médiateur, fonction nouvelle dans ce journal et dans la presse française, trouve naturellement sa place dans la page réservée au courrier. C'est là qu'il s'exprimera chaque semaine pour traiter les questions que se posent les journalistes sur leur travail et, aussi, celles que leur renvoient les lecteurs.

Qu'elles se recoupent, ou qu'elles s'opposent, elles révèlent des tensions, des incompréhensions, des malentendus, des désaccords que l'on saurait laisser en l'état: mieux vaut s'en expliquer franchement, en s'appliquant la transparence que Le Monde exige volontiers des autres. Le médiateur s'y emploiera de son mieux. Il le fera en répondant personnellement à tous ceux qui nous écrivent et, pour les questions d'intérêt général, dans les colonnes du journal.

Dans la première intervention, il précise comment il conçoit cet échange avec les lecteurs, et il entre dans le vif du sujet en abordant, à partir d'une appréciation critique, portée sur notre couverture de l'actualité, le problème de la bonne distance à tenir sur notre couverture de l'actualité, le problème de la bonne distance à tenir dans la relation des évènements et leur évaluation... C'est l'occasion il y a en aura d'autres d'exposer notre conception de l'information en la confrontant aux exigences de nos lecteurs qui sont, eux aussi, partie prenante du Monde.

\section{L'avis du médiateur L'Échange (02/04/1994)}

Le rôle du médiateur, tel que l'a créé la nouvelle direction du Monde, reste largement à inventer. Nous allons nous y employer. Nous, c'est-à-dire tous ceux qui font ce journal et tous ceux qui le Usent. Que les uns et les autres forment, des exemples de cette fonction dans la presse étrangère: au Washington Post, par exemple, ainsi que dans d'autres journaux américains, et, plus près de nous et du modèle que nous envisageons, chez notre confrère espagnol El Païs, avec le titre d'omuswoman car l'actuelle titulaire est une ancienne rédactrice en chef. 
Il existe aussi et c'est le plus important un besoin, sinon un vide, que nous avons ressenti à plusieurs reprises ces dernières années. Un besoin à la fois naturel et étrange pour un journal: celui de communiquer autrement, ou un peu plus que nous le faisons quotidiennement et à sens unique, avec nos lecteurs et, au-delà d'eux, avec les usagers des médias en général.

En vérité, ce lien n'était pas totalement absent. Depuis sa fondation, il y a aura cinquante ans à la fin de l'année, le Monde a reçu, sans doute plus que d'autres journaux en raison de la composition de son lectorat, in courrier abondant. Non seulement les lecteurs nous écrivent, mais ils le font souvent avec une pertinence dérangeante, dans la mesure de ses moyens, le Monde s'est efforcé de maintenir cette relation privilégiée et d'en tenir compte dans sa politique rédactionnelle. Si un besoin nouveau est apparu, c'est plutôt dans la nécessité de donner plus de cohérence et de réciprocité à cette relation et d'en faire le vecteur d'un supplément de dialogue.

2 Le paradoxe de la communication est qu'en s'élargissant tous azimuts, en fondant son essor sur de puissants moyens technologiques, en devenant une production de plus en plus marchande et un enjeu de pouvoir, elle est moins bien reçue et perd de son crédit. La crise de confiance, ou la tentation de la méfiance, touchent tous les organes de presse alors que, globalement, le traitement de l'information en France s'est nettement amélioré depuis la fin de la seconde guerre mondiale.

Sans doute le progrès n-at-il pas été à la mesure du développement des moyens d'informations de masse, d'une part, et, d'autre part, d'une élévation du niveau culturel du public potentiel, lequel gagnait en capacité critique ou scepticisme chronique ce qu'il perdait en faculté d'adhésion aux espérances collectives et aux engagements politiques. Toujours est-il qu'il nous est arrivé, au Monde, comme cela s'est sûrement produit ailleurs, de nous interroger sur l'exercice de notre profession, la nature de l'information que nous livrons à nos lecteurs, la fonction sociale de ce journal, la vertu démocratique de la presse. Il nous est arrivé aussi que les lecteurs nous posent, plus ou moins directement, ces questions.

94 Puisqu'elles se posent de part et d'autres, souvent à partir du traitement courant de l'actualité, pourquoi ne pas en parler ouvertement? Rien n'assure qu'elles obtiendront des réponses évidentes et définitives mais, au moins, cette volonté de dialogue et de transparence cherchera-t-elle à renforcer le lien de confiance sur lequel repose l'échange en matière d'information, entre ceux qui se proposent de la livrer er ceux qui souhaitent la recevoir.

5 Dans la pratique, le médiateur du Monde sera donc l'interlocuteur privilégié des lecteurs, leur intercesseur au sein du journal pour toute interrogation, incompréhension, plainte ou critique, et réciproquement, le porte-parole des journalistes pour affirmer leur bon droit, exposer leurs difficultés, les contraintes et les limites de leur travail, reconnaitre leurs erreurs et en débattre franchement. Il acceptera la discussion sur la manière dont le journal couvre l'actualité et la commente, chaque fois qu'il s'agira de lever une ambiguïté, de dissiper un malentendu ou de s'expliquer sur un silence incompris.

En plus de la correspondance privée, le courrier des lecteurs continuera d'avoir, chaque semaine, sa place dans le journal ; c'est dans cette page que s'exprimera publiquement le médiateur sur les questions ayant un intérêt général. Avec l'espoir que cet échange, car il s'agit d'abord de cela s'approfondira en devenant un rendez-vous naturel. » 


\section{Glossaire}

\section{BIBLIOGRAPHIE}

BOURDIEU Pierre, Choses dites, Paris, Minuit, 1987.

COURTES Joseph, Du lisible au visible, De Boeck Université, 1995, 283 p.

FONTANILLE Jacques, Sémiotique du visible, des mondes de lumière, PUF, 1995, 200 p.

ZILBERBERG Claude, Raison et poétique du sens, PUF, 1988, 227 p.

\section{NOTES}

1. Les deux articles sont dans l'annexe de cette étude.

2. Un glossaire, à l'annexe de cet article précisera les définitions des différents outils théoriques apparaissant dans l'analyse.

3. La dimension ou catégorie thymique est une dimension au même titre que la dimension pragmatique et cognitive qui rendent compte de l'agir des actants dans le discours. La dimension thymique cherchera à saisir le fonctionnement passionnel des actants, c'est-à-dire leur existence modale rendant compte de l'être du sujet, de son intériorité. 
4. AJ. Greimas développe ce concept dans son ouvrage : Sémiotique des passions. Des états de choses aux état d'âme. Paris, Seuil 1991

5. C'est nous qui soulignons en gras les sémèmes (occurences) qui participent a notre analyse.

6. $\mathrm{p} 101$.

7. Nous empruntons cette notion à J. Fontanille dans : Sémiotique du visible. Des mondes de lumière, Paris, PUF, 1995, p 15.

8. Il s'agit en quelque sorte des tensions qui détermineront au niveau des unités discrètes du discours les quatre modalités que sont le devoir, le vouloir, le pouvoir et le savoir.

\section{RÉSUMÉS}

Cette étude est, par une approche strictement sémiotique, l'appréhension d'une figure particulière - le médiateur - à partir du discours journalistique du "Monde " grâce à deux extraits de presse. Nous montrons la prééminence du statut de sujet pathémique, mettant ainsi en retrait l'ordonnance manipulatoire de ce dernier, au plan de l'énonciation énoncée en particulier.

Using a semiotic method, we tried to approach a specific figure in «le Monde » : the journalistic media-tor. We showed how the discourse is enhancing its pathemic organization in order to keep al a distance the enonciative manipulation.

\section{INDEX}

Mots-clés : agencement pathémique, attente, médiateur, médiation, sujet perceptif, volition

\section{AUTEUR}

\section{MARTINE VERSEL}

Martine Versel est maître de conférences à l'ISIC, Université Michel de MontaigneBordeaux 3. Sémioticienne et membre du groupe de recherche GREC/O. 